\title{
Zinn-Haller arterial ring observed by ICG angiography in high myopia
}

\author{
Kyoko Ohno-Matsui, Soh Futagami, Satoshi Yamashita, Takashi Tokoro
}

\begin{abstract}
Aims-To delineate the entire Zinn-Haller arterial ring angiographically in vivo. Methods-382 highly myopic eyes (210 patients) with refractive errors greater than $-8.25 \mathrm{D}$ were examined using indocyanine green (ICG) videoangiography. A control group of 80 eyes ( 40 patients) had refractive errors within plano $+/-3 \mathrm{D}$.

Results-The Zinn-Haller ring was visible in 206 of 382 highly myopic eyes $(53.9 \%)$ by ICG angiography. Although only a part of the Zinn-Haller ring was visible in 162 of 206 eyes, in the remaining 44 eyes it was observed almost completely around the optic nerve head. No anastomotic channels between lateral and medial short posterior ciliary arteries were filled by ICG angiography. In 22 of the 44 eyes $(50.0 \%)$ the Zinn-Haller ring was supplied by branches of the lateral and medial short posterior ciliary arteries; in seven eyes, it was supplied only by the lateral short posterior ciliary artery; and in seven eyes, it was supplied only by the medial short posterior ciliary artery. In none of the control subjects was the Zinn-Haller ring visible by ICG angiography.

Conclusions-The Zinn-Haller ring observed by ICG angiography was not a complete collateral circle between lateral and medial posterior ciliary arteries. Also, the patterns in supply vessels to the Zinn-Haller ring varied. ICG angiography made possible the detailed observation of the Zinn-Haller ring in human eyes in vivo.

(Brf Ophthalmol 1998;82:1357-1362)
\end{abstract}

Ophthalmology,

School of Medicine,

Tokyo Medical and

Dental University,

Japan

K Ohno-Matsui

S Futagami

S Yamashita

$\mathrm{T}$ Tokoro

Correspondence to: Kyoko Ohno-Matsui, MD Department of

Ophthalmology, Tokyo

Medical and Dental

University, 1-5-45 Yushima,

Bunkyo-ku, Tokyo, Japan.

Accepted for publication 12 May 1998

Since Zinn ${ }^{1}$ and Haller ${ }^{2}$ first reported the existence of the arterial circle (Zinn-Haller arterial ring) situated in human sclera around the optic nerve head in 1755, it has been debated whether the ring actually exists. Although many researchers have paid much attention to its existence, shape, and haemodynamic significance, much about this arterial ring has remained unclear because of the difficulty of observing it with current techniques.

Most techniques used to observe the ZinnHaller arterial ring have been histological examinations $^{34}$ or vascular corrosion casting techniques of human cadaver eyes. ${ }^{5-8}$ The vascular corrosion casting technique is more suitable, because the entire blood vessel structure can be observed three dimensionally. However, some researchers have pointed out that the artificial dilatation of non-functioning collateral channels can occur by injecting plastic material forcefully, producing some nonphysiological conditions. ${ }^{9}{ }^{10}$ Moreover, vascular casting cannot provide information about actual blood flow or the circulatory situation in vivo. Therefore, information from both the three dimensional casting technique and haemodynamic angiographic findings are indispensable in evaluating ocular circulation.

To our knowledge, no one has clearly delineated the entire Zinn-Haller ring angiographically. Conventional fluorescein fundus angiography is unsuitable to observe such deeply situated vessel structures. Even using indocyanine green (ICG) angiography, which is a newly developed technique for the examination of choroidal vessel structure, ${ }^{11}{ }^{12}$ it is difficult to observe the Zinn-Haller ring in or external to the sclera around the optic nerve head in normal human eyes.

However, we noticed that the Zinn-Haller arterial ring could be observed in highly myopic eyes within the large annular crescent around the optic nerve head using ICG angiography. In the present study, we observed the circulation of the Zinn-Haller arterial ring in vivo in many highly myopic patients using ICG angiography, and examined the completion of the circle and the supplying artery of the ZinnHaller ring. We compared these ICG angiographic findings with previous findings reported by vascular casting technique.

\section{Materials and methods}

We performed ICG angiography consecutively in 210 highly myopic patients (382 eyes) in our high myopia clinic, Tokyo Medical and Dental University, between September 1993 and November 1995. All patients had a refractive error greater than $-8.25 \mathrm{D}$. None had a history of prior ocular surgery. Their ages ranged from 8 to 78 years (mean 47.9 years). Their refractive errors were from -8.5 to $-36.0 \mathrm{D}$ (mean $-14.6 \mathrm{D}$ ), and the axial lengths were from 26.5 to $32.4 \mathrm{~mm}$ (mean $29.4 \mathrm{~mm}$ ).

As a control group, 80 eyes of 40 healthy volunteers were examined. Their ages ranged 
Table 1 Age, refractive errors, and axial lengths in highly myopic eyes with Zinn-Haller arterial ring visible or not visible by ICG angiography

\begin{tabular}{llllll}
\hline $\begin{array}{l}\text { Observation of } \\
\text { Zinn-Haller ring }\end{array}$ & $\begin{array}{l}\text { Age (years) } \\
\text { (mean (SD) })\end{array}$ & $p^{\star}$ Value & $\begin{array}{l}\text { Refractive error (D) } \\
\text { (mean (SD)) }\end{array}$ & $p^{\star}$ Value $\begin{array}{l}\text { Axial length (mm) } \\
\text { (mean (SD)) }\end{array}$ & $p^{\star}$ Value \\
\hline Visible & $16-74(47.1(14.0))$ & 0.11 & -8.5 to $-28.0(-15.2(4.6))$ & 0.011 & $25.4-32.4(29.8(1.6))$ \\
Not visible & $8-78(49.1(15.1))$ & & -8.0 to $-36.0(-14.0(4.5))$ & & $25.5-32.4(28.9(1.8))$ \\
\hline
\end{tabular}

${ }^{\star} \mathrm{p}$ Value from Mann-Whitney U test.

from 20 to 78 years (mean 55.2 years). Their refractive errors ranged from +2.5 to $-3.0 \mathrm{D}$ (mean -0.5D). Their axial length measurements were from 22.2 to $24.4 \mathrm{~mm}$ (mean 23.4 $\mathrm{mm}$ ). No eye showed fundus abnormality by ophthalmoscopy.

All patients underwent a general ophthalmological examination including indirect ophthalmoscopy and slit lamp biomicroscopy with spherical $+78 \mathrm{D}$ or $+90 \mathrm{D}$ lens (Nikon, Tokyo, Japan). The determination of the refractive error was based on subjective refraction following the retinoscopy measurement. The refractive error was shown as the spherical equivalent. Axial length measurement with the A mode ultrasonic scan (Alpha 2, Storz, St Louis, MO, USA) was performed in all patients. Colour fundus photography using a fundus camera (Pro 1, Kowa, Tokyo, Japan) was performed in all patients.

After the rapid injection of $50 \mathrm{mg}$ ICG dye into the subject's antecubital vein, we performed ICG videoangiography using the fundus camera (TRC-50IA, Topcon, Tokyo, Japan) and a real time video image quality improvement system (DVS-1000, Hamamatsu Photonics Co, Hamamatsu, Japan). The ICG videoangiographic sequence was videotaped on a videocassette recorder and was observed continuously on a videoscreen. Printed images were obtained by a film recorder (FR-1100, Avio, Tokyo, Japan).

The observation of the Zinn-Haller arterial ring was done in the early phase of the angiogram following the injection of ICG dye for 2 minutes. Then we detected branches of retrobulbar short posterior ciliary arteries that supplied the arterial ring, from the positional relation with choroidal vessels following the ocular movement, as reported previously. ${ }^{13}$ The detection of a supply vessel was done only in highly myopic eyes whose Zinn-Haller ring was visible almost completely around the optic nerve head.

Each angiogram was evaluated in a masked fashion by three of us (KOM, SF, SY). Agreement among the observers regarding shape and supply vessels of the Zinn-Haller arterial ring was obtained in all angiograms. We also performed statistical analysis to find correlat-

Table 2 Short posterior ciliary artery supply to Zinn-Haller ring in 44 eyes whose ring was visible completely around the optic nerve head

\begin{tabular}{lcl}
\hline Type & No of eyes & \% of eyes \\
\hline Medial and lateral SPCAs & 22 & 50.0 \\
Medial SPCA & 7 & 15.9 \\
Lateral SPCA & 7 & 15.9 \\
Unknown & 8 & 21.1 \\
\hline
\end{tabular}

SPCA $=$ short posterior ciliary artery. ing factors on visibility of the Zinn-Haller arterial ring in highly myopic eyes by ICG angiography. The Mann-Whitney $U$ test was adopted to compare age, refractive error, and axial length between the highly myopic eyes whose Zinn-Haller ring was visible or not. A p value less than 0.05 was considered significant.

Informed consent was obtained from all subjects examined. The above examination protocol was approved by the ethics committee of Tokyo Medical and Dental University.

\section{Results}

We observed the Zinn-Haller arterial ring around the optic nerve head in 206 (53.9\%) of 382 highly myopic eyes. Table 1 compares age, axial length, and refractive error in highly myopic patients whose Zinn-Haller arterial ring was visible or not visible. Patients with a visible Zinn-Haller ring were extremely myopic. They were more highly myopic and had longer axial lengths than patients whose arterial ring was not visible.

Where the Zinn-Haller ring was visible depended on the type of peripapillary crescent. In $153(74.3 \%)$ of 206 eyes, only a temporal part of the Zinn-Haller ring was observed through the temporal crescent of the optic nerve head. In nine (4.4\%) of the 206 eyes, only inferior parts of the Zinn-Haller ring were visible. These nine eyes had an inferior crescent accompanied by a tilted disc. In the remaining 44 eyes (21.4\%), the Zinn-Haller ring was observed almost completely around the optic nerve head (longer than three quarters of the surroundings of the optic nerve head). These eyes had an annular crescent around the optic nerve head.

We detected the supply vessel to the Zinn-Haller ring in these 44 eyes (Table 2). Representative figures of these 44 eyes are shown in Figure 1-5. In $22(50.0 \%)$ of 44 eyes, the Zinn-Haller ring was supplied by branches of both the lateral and medial short posterior ciliary arteries (Fig 2). In seven eyes (15.9\%), it was supplied only by the medial short posterior ciliary artery (Figs 3 and 4). In seven eyes $(15.9 \%)$, it was supplied only by the lateral short posterior ciliary artery (Fig 5). In the remaining eight eyes (18.2\%), the supplying artery was not clear because the images of the retrobulbar part of the short posterior ciliary arteries were not obvious.

None of the Zinn-Haller rings in these 44 eyes formed a complete circle. In all cases, the Zinn-Haller ring was not continuous in the superior or inferior part of the optic nerve head. No anastomotic channels between lateral and medial short posterior ciliary arteries were filled 

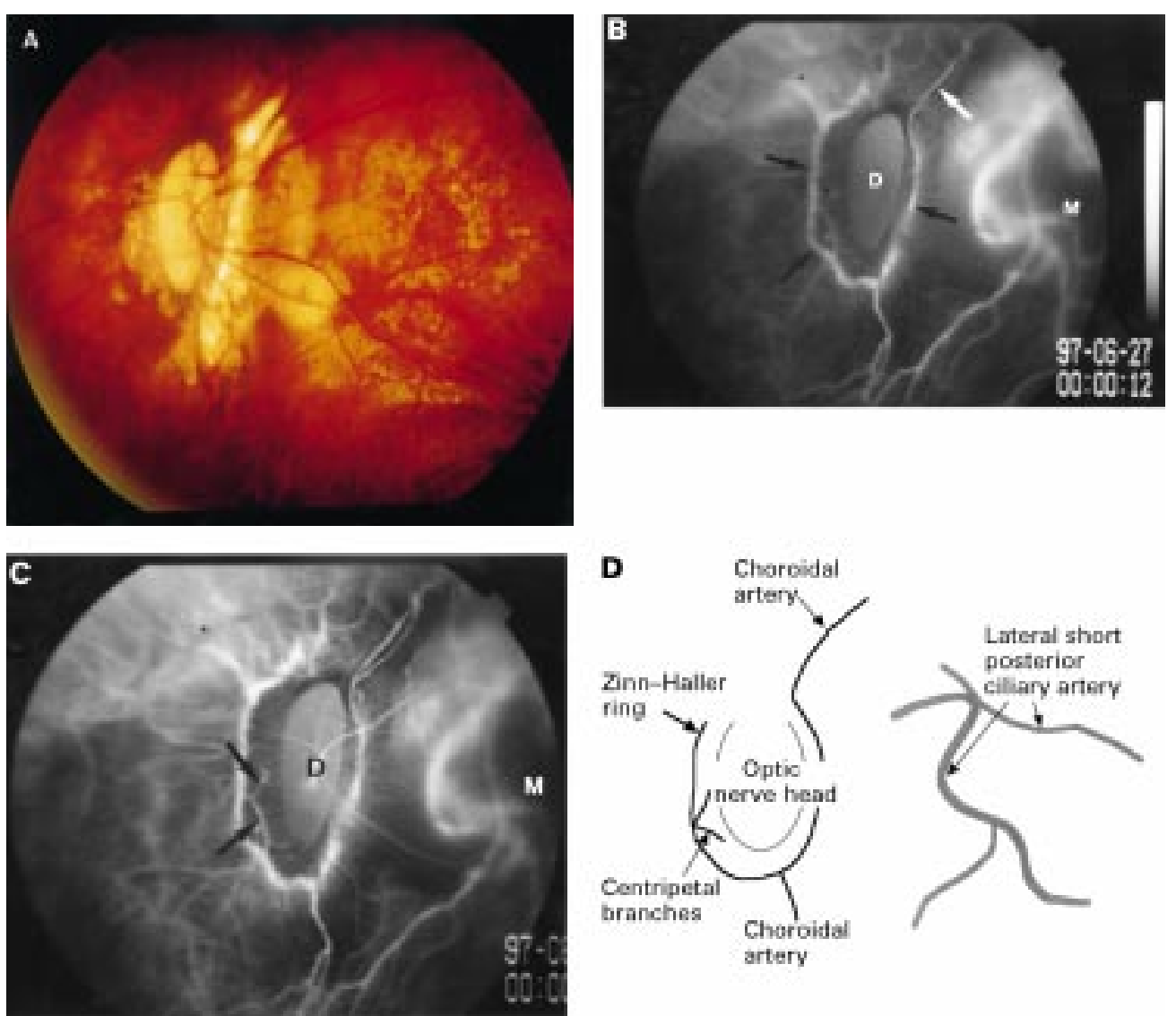

Figure 1 Typical case with visible Zinn-Haller ring. (A) Left fundus shows an annular crescent around the optic nerve head and diffuse chorioretinal atrophy in the posterior fundus. (B) In the angiogram 12 seconds after ICG dye injection, the incomplete Zinn-Haller ring (black arrows) is observed around the optic nerve head. It is continuous with a peripapillary choroidal artery (white arrow) in the superior part of the optic nerve head. $D=$ optic nerve head, $M=$ macula. (C) At 14 seconds after ICG dye injection, centripetal branches towards the optic nerve head (arrows) originating from the Zinn-Haller ring are observed. (D) Schematic drawing of the angiogram seen in (B).

by ICG angiography. On the other hand, the Zinn-Haller ring was not visible in any of the control subjects even using ICG angiography.

\section{Discussion}

In 1978, Hayreh ${ }^{13}$ examined 106 human cadaver eyes histologically and concluded that the Zinn-Haller ring was rarely observed, and when it existed, it usually was an incomplete circle. After this description, many researchers doubted the existence or the significance of the Zinn-Haller ring in optic nerve circulation.

With the recent development of vascular casting techniques using methyl methacrylate, it has become possible to produce high quality corrosion casts of the vascular structure in human cadaver eyes. Using this technique, many new findings have been made. Awai ${ }^{5}$ examined nine human cadaver eyes and found that the Zinn-Haller ring existed in all the eyes he examined. Olver et al ${ }^{7}$ examined 18 human cadaver eyes using vascular casting technique and observation by scanning electron microscopy, and also found the Zinn-Haller ring in all examined eyes. Moreover, the authors ${ }^{7}$ described that a complete Zinn-Haller ring was found in 14 of 18 examined eyes (77.0\%), including anastomoses with narrow portions in the superior and inferior parts of the optic nerve head. These recent findings ${ }^{5-8}$ obtained by vascular casting technique and scanning electron microscopy have shown the ZinnHaller ring to be a common vascular structure in human eyes. That ring is also considered to be a complete circle in most cases. Within this arterial ring, branches of lateral and medial short posterior ciliary arteries directly connect with each other or through narrow anastomotic channels in the superior and inferior part of the optic nerve head.

The vascular casting technique is suitable to observe the Zinn-Haller ring and its supplying vessel in a complete and three dimensional way. However, even in highly elaborated corrosion casts, some researchers ${ }^{9}{ }^{10}$ have suggested that results may be misinterpreted. The plastic material used in the corrosion cast is very different from blood itself in nature. Even if the pressure is measured while the material is injected, potential anastomotic vessels (that do not function in physiological conditions) might dilate. ${ }^{9}{ }^{10}$ Therefore, it is dangerous to evaluate the ocular circulation solely by the results obtained using the vascular casting technique. We believe that angiographic findings in human eyes in vivo add indispensable information to evaluate the blood flow and circulation of the Zinn-Haller ring.

ICG angiography has several advantages in examining the Zinn-Haller ring. Firstly, it can be performed easily in many human subjects in vivo, unlike the limitation of using human 


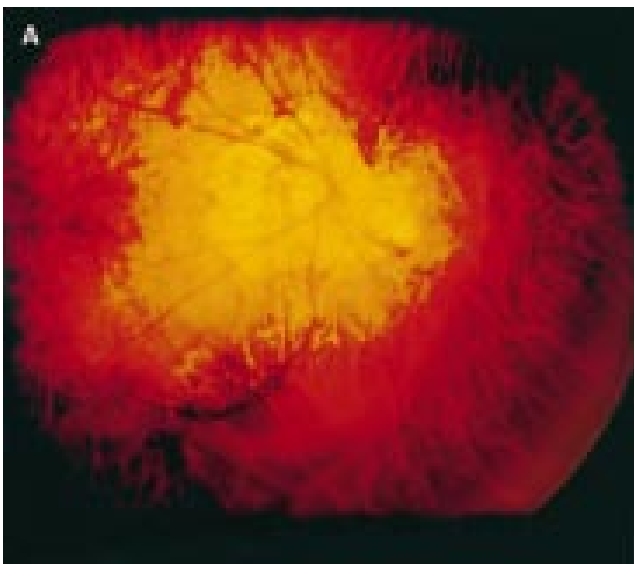

Figure 2 Zinn-Haller ring supplied by branches of both lateral and medial short posterior ciliary arteries. (A) Right fundus shows temporal crescent around the optic nerve head and diffuse chorioretinal atrophy in the posterior fundus. (B) In the ICG angiogram 16 seconds after dye injection, rhomboid-shaped Zinn-Haller ring (arrowheads) is visible around the optic nerve head. The temporal part of the ring seems to be continuous with the lateral short posterior ciliary artery. The nasal part of the ring originates from one branch of the medial short posterior ciliary artery (black arrow). Centripetal branches (white arrows) towards the optic nerve head are observed. $D=$ optic nerve head, $M=$ macula. (C) Schematic drawing of the angiogram seen in $(B)$.

cadaver eyes. Secondly, ICG angiography enables the clear observation of the blood flow to the Zinn-Haller ring in vivo although restricted in highly myopic eyes. Thirdly, in ICG angiograms of highly myopic patients, retrobulbar vascular structure is also visible, ${ }^{14}$ revealing which branches of retrobulbar short posterior ciliary arteries are actually involved in

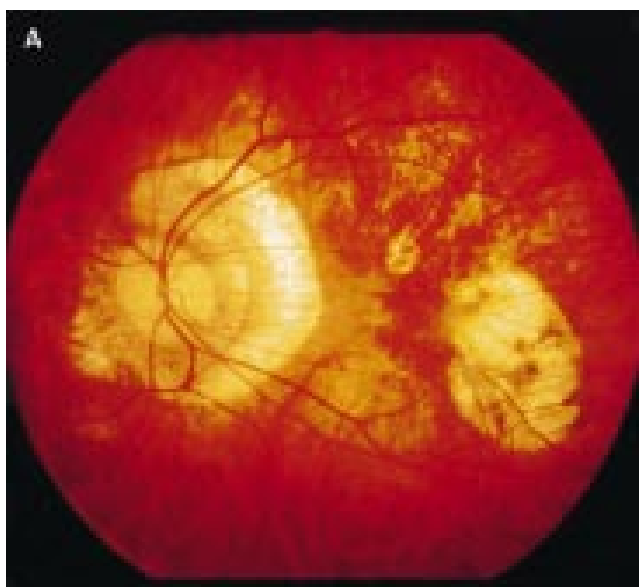

Figure 3 Zinn-Haller ring supplied only by the medial short posterior ciliary artery. (A) Left fundus shows large annular crescent around the optic nerve head. (B) At 16 seconds after dye injection, the Zinn-Haller ring (white arrowheads) is completely filled by ICG dye. The nasal part of Zinn-Haller ring originates from one branch (white arrows) of medial short posterior ciliary artery. Careful observation shows temporal part of Zinn-Haller ring also originates from one branch (black arrowheads) of the medial short posterior ciliary artery. Lateral short posterior ciliary artery is not involved in blood supply to Zinn-Haller ring in this patient. The Zinn-Haller ring is not a complete circle. In the superior part of the optic nerve head, the nasal part of the Zinn-Haller ring is continuous with peripapillary choroidal artery (black arrows). $D=$ optic nerve head, $M=$ macula. (C) Schematic drawing of the angiogram seen in $(B)$.

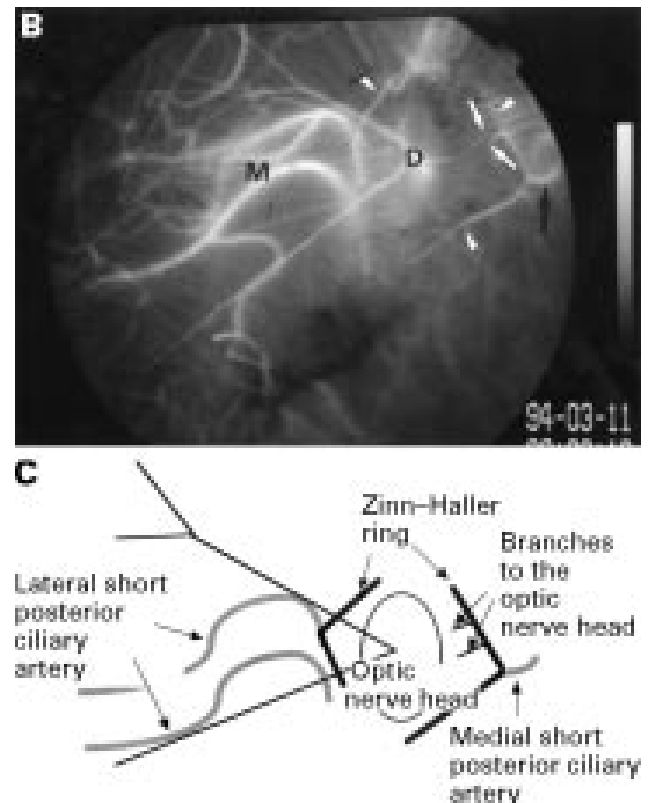

the blood supply of the Zinn-Haller ring haemodynamically.

Ko et $a l^{15}$ recently described fluorescein angiographical findings of the Zinn-Haller ring in 15 highly myopic eyes, but were unable to demonstrate complete figures of the ring by fluorescein angiography. Also, only one case report described the observation of the Zinn-

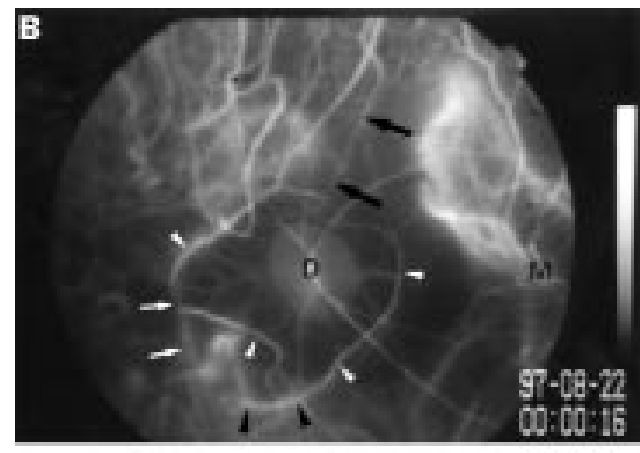

\section{C}

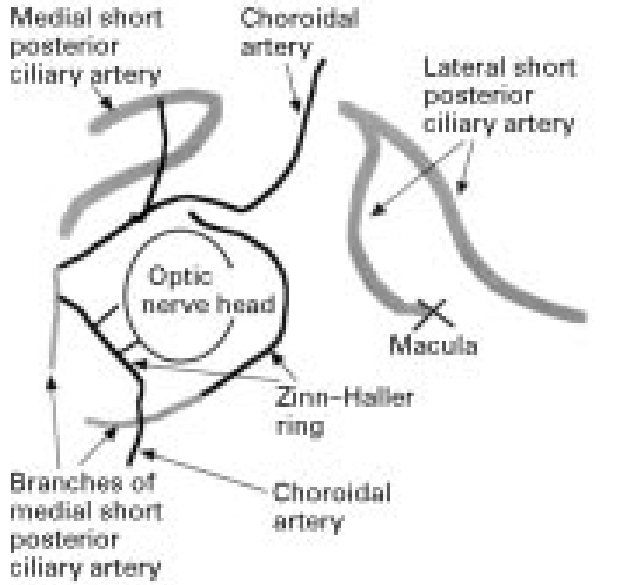




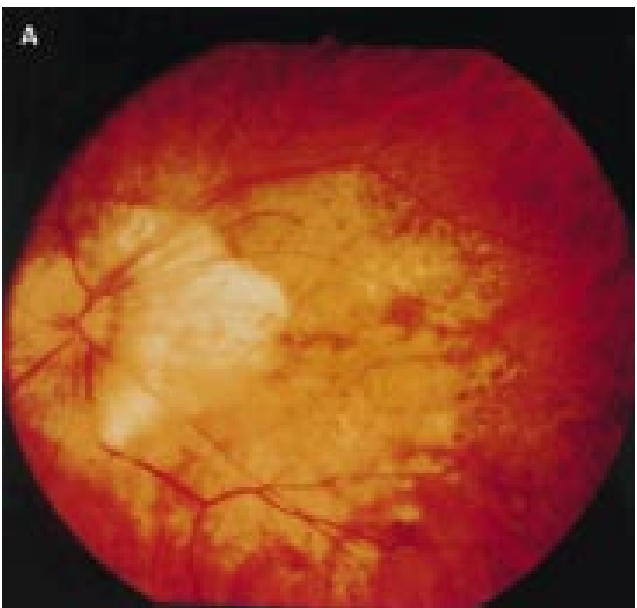

Figure 4 Zinn-Haller ring supplied only by the medial short posterior ciliary artery. (A) Left fundus shows large temporal crescent around the optic nerve head. (B) At 20 seconds after dye injection, the Zinn-Haller ring (white arrowheads) is filled by ICG dye. The Zinn-Haller ring originates from medial short posterior ciliary artery (white arrows). Lateral short posterior ciliary artery (black arrowheads) is not involved in blood supply to Zinn-Haller ring in this patient. The Zinn-Haller ring is not a complete circle. In the superior part of the optic nerve head, the Zinn-Haller ring is continuous with peripapillary choroidal artery (black arrow). $D=$ optic nerve head, $M=$ macula. (C) Schematic drawing of the angiogram seen in (B).

Haller ring using ICG angiography by Park et $a l^{16}$ However, in their case, only the temporal part of the ring was visualised.

In this study, we observed the Zinn-Haller ring in 206 of 382 eyes (53.9\%) using ICG angiography. In 153 eyes, only the temporal part of the Zinn-Haller ring was observed, because myopic crescents are usually located temporal to the optic nerve head in highly myopic eyes. Such cases are not suitable for further examination. We next selected 44 eyes whose Zinn-Haller ring was visible almost completely around the optic nerve head to

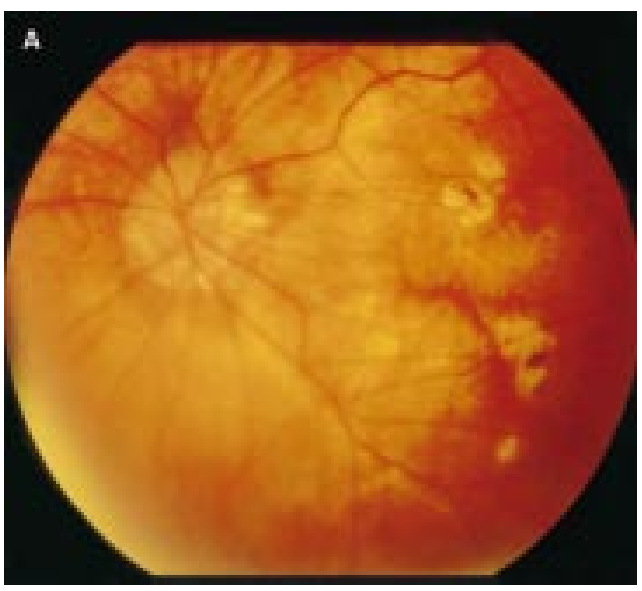

Figure 5 Zinn-Haller ring supplied only by the lateral short posterior ciliary artery. (A) Left fundus shows an annular crescent around the optic nerve head and diffuse chorioretinal atrophy in the posterior fundus. (B) In the ICG angiogram 20 seconds after dye injection, the intense fluorescence due to lateral short posterior ciliary artery (white arrows) and the Zinn-Haller ring are observed (arrowheads). The Zinn-Haller ring seems to branch from the lateral short posterior ciliary artery (black arrow). $D=$ optic nerve head, $M=$ macula. (C) Schematic drawing of the angiogram seen in (B).
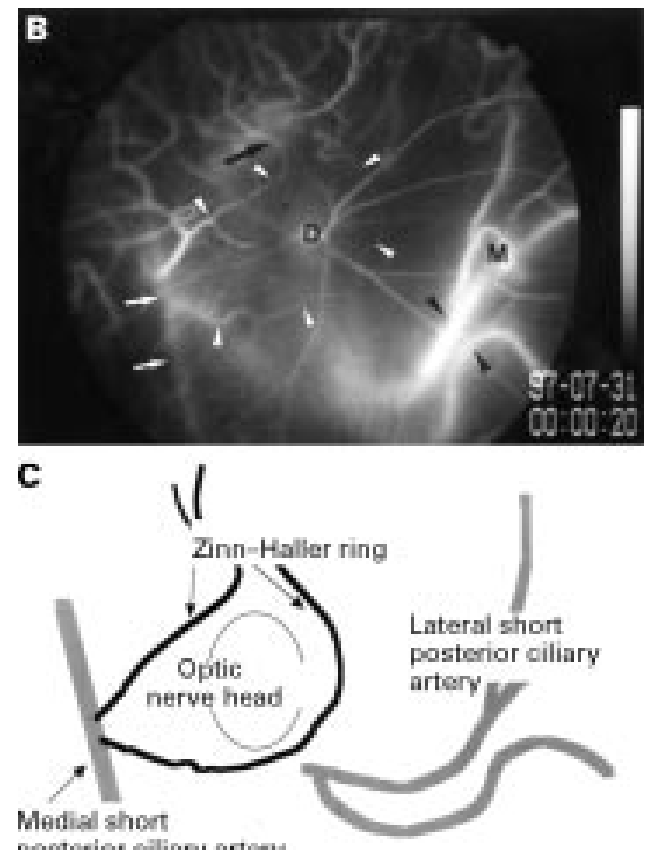

posterior ciliary artery

determine the completion and supply vessel of this arterial ring.

Most of these 44 eyes had an annular crescent around the optic nerve head. No Zinn-Haller ring in these 44 eyes was a complete circle. A narrow anastomotic channel in the superior and inferior parts of the optic nerve head, as described by Olver et al, ${ }^{7}$ was not observed. From these findings, the ZinnHaller ring was found not to function as a complete circle in vivo, at least in highly myopic eyes. There still is a possibility that missing narrow anastomotic channels between
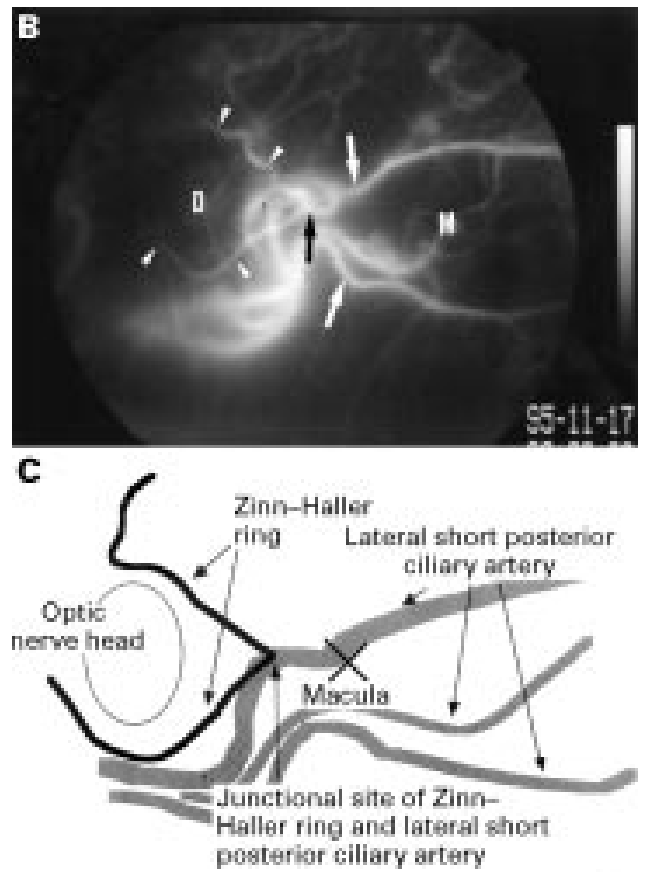
lateral and medial short posterior ciliary arteries may be present, because of low resolution of ICG angiography. However, we consider that the narrow anastomotic channel between lateral and medial short posterior ciliary arteries described by Olver et al may have been a potential anastomotic channel that might function in some pathological conditions, such as the occlusion of medial or lateral short posterior ciliary artery.

For a long time, the Zinn-Haller ring has been regarded as an anastomotic circle between the lateral and medial short posterior ciliary arteries. However, Olver et al first reported three different types of blood supply patterns of the Zinn-Haller ring using the vascular casting technique. One pattern is supplied by both lateral and medial short posterior ciliary arteries and was observed in 15 of 18 eyes $(83.0 \%)$. One is supplied by three short posterior ciliary arteries-lateral, medial and superior-and was observed in two of 18 eyes $(11.0 \%)$. The last is supplied only by the lateral short posterior ciliary artery. This pattern was observed in only one of 18 eyes (5.6\%). Olver et $a l$ suggested that eyes whose Zinn-Haller ring is supplied by only one short posterior ciliary artery are vulnerable to anterior optic nerve ischaemia.

In the present study, 14 of 44 eyes (31.8\%) had a Zinn-Haller ring that was supplied by only one lateral or medial short posterior ciliary artery (Table 2). Although there remains a possibility of the contribution of both lateral and medial short posterior ciliary arteries even in eyes that show one artery's contribution by ICG angiography, this finding showed that the rings supplied by only one short posterior ciliary artery might be more common than expected.

Moreover, in seven of these 14 eyes, the ring was supplied only by the medial short posterior ciliary artery. To our knowledge, no authors have reported this type of blood supply to the Zinn-Haller ring. It remains unclear whether this pattern is present in only highly myopic eyes or may also exist in normal eyes.
This study showed that ICG angiography made it possible to observe the Zinn-Haller ring in a large number of human eyes in vivo, although the present method of ICG angiography still has some limitations and is restricted in extremely myopic eyes. ICG angiography could be used to evaluate not only the choroidal circulation but also the circulation of the optic nerve.

This work was supported by grant in aid for Scientific Research No A199 from the Ministry of Education, Science and Culture of Japan and by a grant for Retinochoroidal Atrophy Research from the Ministry of Health and Welfare of Japan.

1 Zinn JG. Descriptio anatomica oculi humani. Gottigen, 1755. Cited by Francois et al in Br 7 Ophthalmol 1954; 1755. Cited
38:472-88.

2 Haller A. Arteriarum oculi historia et tabulae arteriarum oculi. Gottigen, 1754. Cited by Francois et al in $\mathrm{Br} \mathcal{F} O \mathrm{ph}$ thalmol 1954;38:472-88

3 Levitzky M, Henkind P. Angioarchitecture of the optic nerve-lamina cribrosa. Am f Ophthalmol 1969;68:986-96.

4 Liebermann MF, Maumenee AE, Green WR. Histologic study of the vasculature of the anterior optic nerve. Am $\mathcal{F}$ Ophthalmol 1976;82:405-23.

5 Awai T. Angioarchitecture of intraorbital part of human optic nerve. Ipn f Ophthalmol 1985;29:79-98.

6 Olver JM, Spalton DJ, McCartney ACE. Microvascular study of the retrolaminar optic nerve in man: the possible significance in anterior ischemic optic neuropathy. Eye 1990;4:7-24.

7 Olver JM, Spalton DJ, McCartney ACE. Quantitative morphology of human retrolaminar optic nerve vasculature. phology of human retrolaminar optic nerve

8 Zhao Y, Fengming LI. Microangioarchitecture of optic papilla. Fpn f Ophthalmol 1987;31:147-59.

9 Araki M. Ciliary vein in monkey. Acta Soc Ophthalmol fap 1975;79:590-3.

10 Yoshimoto H, Murata M, Yamaguchi K, et al. Studies on the angioarchitecture of the posterior choroid in rat and role of posterior ciliary vein. Invest Ophthalmol Vis Sci 1980;19: $1245-50$.

11 Flower RW, Hochheimer BF. A clinical technique and apparatus for simultaneous angiography of the separate retinal and choroidal circulations. Invest Ophthalmol Vis Sci 1973; 12:248-61.

12 Hayashi K, Hasegawa Y, Tazawa Y. Clinical application of indocyanine green angiography to choroidal neovasculariindocyanine green angiography to choro

13 Hayreh SS. Structure and blood supply of the optic nerve. In: Heilmann K, ed. Glaucoma, concepts of a diseasepathogenesis. Stuttgart: Georg Thieme, 1978:78-84.

14 Ohno-Matsui K, Morishima N, Ito M, et al. Indocyanine green fundus angiography of retrobulbar vascular structure in high myopia. Am f Ophthalmol 1997;123:494-505.

15 Ko MK, Kim DS, Ahn YK. Peripapillary circle of Zinn-Haller revealed by fundus fluorescein angiography. $\mathrm{Br}$ f Ophthalmol 1997;81:663-7.

16 Park KH, Tomita G, Onda E, et al. In vivo detection of perineural circular arterial anastomosis (circle of ZinnHaller) in a patient with large peripapillary chorioretinal atrophy. Am f Ophthalmol 1996;122:905-7. 